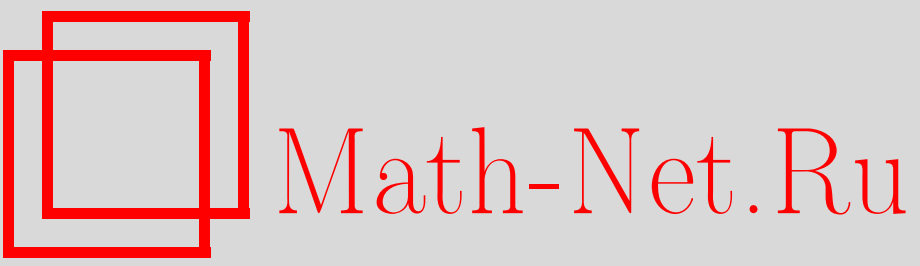

С. В. Козырев, Оснащенное гильбертово пространство свободных когерентных состояний и $p$-адические числа, ТМФ, 2003, том 135, номер 2, 229-239

DOI: https://doi.org/10.4213/tmf186

Использование Общероссийского математического портала Math-Net.Ru подразумевает, что вы прочитали и согласны с пользовательским соглашением

http://www.mathnet.ru/rus/agreement

Параметры загрузки:

IP : 35.173 .219 .149

26 апреля 2023 г., 16:50:45 
ТЕОРЕТИЧЕСКАЯ

И МАТЕМАТИЧЕСКАЯ

ФИЗИКА

Том 135, № 2

май, 2003

(C) 2003 г.

С. В. Козырев*

\section{ОСНАЩЕННОЕ ГИЛЬБЕРТОВО ПРОСТРАНСТВО СВОБОДНЫХ КОГЕРЕНТНЫХ СОСТОЯНИЙ И $p$-АДИЧЕСКИЕ ЧИСЛА}

Исследовано оснащенное гильбертово пространство свободных когерентных состояний. Доказано, что это оснащенное гильбертово пространство изоморфно пространству обобщенных функций на $p$-адическом диске. Обсуждается связь описанного изоморфизма оснащенных гильбертовых пространств и некоммутативной геометрии. Показано, что обсуждаемый пример реализует изоморфизм между некоммутативной прямой и $p$-адическим диском.

Ключевые слова: -адические числа, некоммутативная геометрия.

\section{1. ВВЕДЕНИЕ}

В настоящей работе, продолжая исследования [1], [2], мы рассматриваем свободные когерентные состояния (СКC), являющиеся (неограниченными) собственными векторами линейной комбинации операторов уничтожения в свободном фоковском пространстве. В работах [1], [2] было показано, что пространство СКС сильно вырожденно для фиксированного собственного значения $\lambda$ (вырождение бесконечномерно) и это вырождение естественно описывается пространством $D^{\prime}\left(\mathbb{Z}_{p}\right)$ обобщенных функций на $p$-адическом диске (здесь $p$ - количество независимых операторов рождения в свободном фоковском пространстве). В настояшей работе мы переформулируем результаты [1], [2], используя язык оснашенных гильбертовых пространств, и предлагаем интерпретацию связи между $\mathrm{CKC}$ и $p$-адическими числами на языке некоммутативной геометрии. На этом языке изоморфизм меж ду пространством СКС и пространством обобщенных функций на $p$-адическом диске сводится к изоморфизму между некоммутативной (или квантовой) прямой и $p$-адическим диском.

$p$-Адическая математическая физика изучает проблемы математической физики при помоши $p$-адического анализа [3]-[10]. В частности, в книге [3] был развит анализ $p$-ади-

${ }^{*}$ Институт химической физики РАН им. Н. Н. Семенова, Москва, Россия. E-mail: kozyrev@mi.ras.ru 
ческих псевдодифференциальных операторов. В работе [4] был предложен $p$-адический подход в теории струн. В [6] была развита теория $p$-адичнозначных обобщенных функций. В работах [8] было показано, что матрица Паризи, используемая в методе реплик, в простейшем случае эквивалентна $p$-адическому псевдодифференциальному оператору. В [9] показано, что базис всплесков (или вейвлетов) в пространстве $L^{2}(\mathbb{R})$ после $p$-адической замены переменной (непрерывного отображения $p$-адических чисел на вешественные числа, сохраняюшего меру) становится базисом собственных векторов оператора Владимирова $p$-адического дробного дифференцирования. В работе [10] была предложена процедура, порождающая ультраметрическое пространство состояний, используемое в методе реплик.

Свободное (или квантовое больцмановское) фоковское пространство рассматривалось в работах по квантовой хромодинамике [11] и некоммутативной вероятности [12].

Настояшая работа имеет следуюшую структуру. В разделе 2 вводятся СКС. В разделе 3 обсуждается изоморфизм меж ду СКС и обобщенными функциями на $p$-адическом диске. В разделе 4 на пространстве СКС вводится структура оснашенного гильбертова пространства и изоморфизм, обсуждаемьй в разделе 3 , обобшается на случай оснащенных гильбертовых пространств.

\section{2. СВОБОДНЫЕ КОГЕРЕНТНЫЕ СОСТОЯНИЯ}

Свободное фоковское пространство $\mathcal{F}$ над гильбертовым пространством $\mathcal{H}$ есть пополнение тензорной алгебры

$$
\mathcal{F}=\bigoplus_{n=0}^{\infty} \mathcal{H}^{\otimes n} .
$$

Операторы рождения и уничтожения действуют следующим образом:

$$
\begin{aligned}
A^{\dagger}(f) f_{1} \otimes \cdots \otimes f_{n} & =f \otimes f_{1} \otimes \cdots \otimes f_{n}, \quad f, f_{i} \in \mathcal{H} \\
A(f) f_{1} \otimes \cdots \otimes f_{n} & =\left\langle f, f_{1}\right\rangle f_{2} \otimes \cdots \otimes f_{n}, \quad f, f_{i} \in \mathcal{H}
\end{aligned}
$$

где $\langle\cdot, \cdot \cdot\rangle$ есть скалярное произведение в гильбертовом пространстве $\mathcal{H}$. Скалярное произведение в свободном фоковском пространстве (которое мы также обозначаем $\langle\cdot, \cdot\rangle$ ) определяется стандартным способом.

В случае, когда $\mathcal{H}$ является $p$-мерным комплексным пространством, имеются по $p$ операторов рождения и уничтожения $A_{i}^{\dagger}, A_{i}, i=0, \ldots, p-1$, удовлетворяюших соотношениям

$$
A_{i} A_{j}^{\dagger}=\delta_{i j}
$$

и вакуумный вектор $\Omega$ в свободном фоковском пространстве: $A_{i} \Omega=0$.

СКС были введены в работах [1], [2] как формальные собственные векторы оператора уничтожения $A=\sum_{i=0}^{p-1} A_{i}$ в свободном фоковском пространстве $\mathcal{F}$ для некоторого собственного значения $\lambda$,

$$
A \Psi=\lambda \Psi .
$$


Формальное решение уравнения (2) имеет вид

$$
\Psi=\sum_{I} \lambda^{|I|} \Psi_{I} A_{I}^{\dagger} \Omega
$$

Здесь мультииндекс есть $I=i_{0} \ldots i_{k-1}, i_{j} \in\{0, \ldots, p-1\}$, и

$$
A_{I}^{\dagger}=A_{i_{k-1}}^{\dagger} \ldots A_{i_{0}}^{\dagger} \text {. }
$$

Коэффициенты $\Psi_{I}$ суть комплексные числа, удовлетворяюшие

$$
\Psi_{I}=\sum_{i=0}^{p-1} \Psi_{I i}
$$

Суммирование в формуле (3) производится по всем последовательностям $I$ конечной длины. Длина последовательности $I$ обозначается $|I|$ (в частности, в формуле (4) $|I|=k)$. Формальный ряд (3) определяет функционал с плотной областью определения в свободном фоковском пространстве. В частности, область определения каждого СКС для $\lambda \in(0, \sqrt{p})$ содержит плотное (в свободном фоковском пространстве) подпространство $X$, введенное ниже.

Введем CKC $X_{I}$ вида

$$
X_{I}=\sum_{k=0}^{\infty} \lambda^{k}\left(\frac{1}{p} \sum_{i=0}^{p-1} A_{i}^{\dagger}\right)^{k} \lambda^{|I|} A_{I}^{\dagger} \Omega+\sum_{l=1}^{\infty} \lambda^{-l}\left(\sum_{i=0}^{p-1} A_{i}\right)^{l} \lambda^{|I|} A_{I}^{\dagger} \Omega .
$$

Сумма по $l$ фактически содержит $|I|$ членов. Для $\lambda \in(0, \sqrt{p})$ CKC $X_{I}$ лежит в гильбертовом пространстве (соответствуюший функционал ограничен).

Обозначим через $X$ линейную оболочку CKC вида (6), а через $X^{\prime}$ - пространство всех CKC (заданных в (3)).

Определение 1 было предложено, а леммы 1,2 и 4, следствие 1 и пример (см. ниже) были доказаны в работах [1], [2].

ОПРЕДЕЛЕниЕ 1. Определим перенормированное спаривание пространств $X$ и $X^{\prime}$ следуюшим образом:

$$
(\Psi, \Phi)=\lim _{\lambda \rightarrow \sqrt{p}-0}\left(1-\frac{\lambda^{2}}{p}\right)\langle\Psi, \Phi\rangle,
$$

где $\Psi \in X^{\prime}, \Phi \in X$.

Отметим, что когерентные состояния $\Psi, \Phi$, определенные формулами (3), (6), зависят от $\lambda$, а произведение $(\Psi, \Phi)$ от $\lambda$ не зависит.

Корректность определения 1 доказывается следующей леммой. 
Лемма 1. Векторы $X_{I} \in X$ принадлежсат области определения функиионала $\Psi$ для $\lambda \in(0, \sqrt{p})$ и для произвольного $C K C \Psi$, определенного формулой (3). Более того, следующий предел существует и равен

$$
\left(\Psi, X_{I}\right)=\lim _{\lambda \rightarrow \sqrt{p}-0}\left(1-\frac{\lambda^{2}}{p}\right)\left\langle\Psi, X_{I}\right\rangle=p^{|I|} \Psi_{I} .
$$

ДокАЗАТЕльство. Спаривание функционала $\Psi \in X^{\prime}$, заданного в $(3)$, и состояния (6) задается следующим рядом:

$$
\left\langle\Psi, X_{I}\right\rangle=\sum_{k=0}^{\infty} \lambda^{2 k}\left\langle\Psi^{k}, X_{I}^{k}\right\rangle
$$

Здесь $\Psi^{k}$ и $X_{I}^{k}-$ коэффициенты при $\lambda^{k}$ в рядах для $\Psi$ и $X_{I}$. Вектор $\Psi^{k}$ задан формулой

$$
\Psi^{k}=\sum_{|J|=k} \Psi_{J} A_{J}^{\dagger} \Omega
$$

и $X_{I}^{k}$ для $k>|I|$ имеет вид

$$
X_{I}^{k}=\left(\frac{1}{p} \sum_{i=0}^{p-1} A_{i}^{\dagger}\right)^{k-|I|} A_{I}^{\dagger} \Omega
$$

Для $k>0$ мы получим

$$
\begin{aligned}
\left\langle\Psi^{|I|+k}, X_{I}^{|I|+k}\right\rangle & =\left\langle X_{I}^{|I|+k}, \Psi^{|I|+k}\right\rangle^{*}=\left\langle X_{I}^{|I|+k-1}, \frac{1}{p} \sum_{i=0}^{p-1} A_{i} \Psi^{|I|+k}\right\rangle^{*}= \\
& =\left\langle X_{I}^{|I|+k-1}, \frac{1}{p} \sum_{i=0}^{p-1} A_{i} \sum_{|J|=|I|+k} \Psi_{J} A_{J}^{\dagger} \Omega\right\rangle^{*} .
\end{aligned}
$$

Отсюда следует

$$
\frac{1}{p} \sum_{i=0}^{p-1} A_{i} \sum_{|J|=|I|+k} \Psi_{J} A_{J}^{\dagger} \Omega=\sum_{|J|=|I|+k-1} \frac{1}{p} \sum_{i=0}^{p-1} \Psi_{J i} A_{I}^{\dagger} \Omega=\frac{1}{p} \Psi^{|I|+k-1}
$$

Следовательно,

$$
\left\langle\Psi^{|I|+k}, X_{I}^{|I|+k}\right\rangle=\frac{1}{p}\left\langle\Psi^{|I|+k-1}, X_{I}^{|I|+k-1}\right\rangle=p^{-k}\left\langle\Psi^{|I|}, X_{I}^{|I|}\right\rangle=p^{-k} \Psi_{I},
$$

откуда получаем, что ряд (9) принимает вид

$$
\left\langle\Psi, X_{I}\right\rangle=\sum_{k=0}^{|I|} \lambda^{2 k}\left\langle\Psi^{k}, X_{I}^{k}\right\rangle+\sum_{k=|I|+1}^{\infty} \lambda^{2 k} p^{|I|-k} \Psi_{I}
$$

Поскольку для $\lambda^{2} / p<1$ вышеприведенный ряд мажорируется геометрической прогрессией, ряд (9) сходится и соответствующее перенормированное спаривание принимает Вид

$$
\left(\Psi, X_{I}\right)=p^{|I|} \Psi_{I}
$$

Это завершает доказательство леммы. 


\section{3. СВЯЗЬ С $p$-АДИЧЕСКИМИ ЧИСЛАМИ}

Определим характеристическую функцию $p$-адического диска

$$
\theta_{k}\left(x-x_{0}\right)=\theta\left(p^{k}\left|x-x_{0}\right|_{p}\right), \quad \theta(t)=\left\{\begin{array}{l}
0, t>1 \\
1, t \leqslant 1
\end{array}\right.
$$

Здесь $x, x_{0} \in \mathbb{Z}_{p}$, т.е. принадлежат кольцу целых $p$-адических чисел, и функция $\theta_{k}(x-$ $\left.x_{0}\right)$ равна единице на диске $D\left(x_{0}, p^{-k}\right)$ радиуса $p^{-k}$ с центром в $x_{0}$ и равна нулю вне этого диска.

Отождествим мультииндекс $I=i_{0} \ldots i_{k}$ с $p$-адическим числом $I=\sum_{j=0}^{k} i_{j} p^{j}$.

Следуюшая лемма описывает отношение между перенормированным спариванием СКС и скалярным произведением квадратично-интегрируемых функций на $p$-адическом диске.

ЛЕмма 2. Пространство $X$ с перенормированным скалярным произведением изоморфно, как евклидово пространство, пространству $D\left(\mathbb{Z}_{p}\right)$ основных функиий на р-адическом диске со скалярным произведением в $L^{2}$, причем изоморфизм задан как

$$
\begin{gathered}
\phi: X \rightarrow D\left(\mathbb{Z}_{p}\right), \\
\phi: X_{I} \mapsto p^{|I|} \theta_{|I|}(x-I) .
\end{gathered}
$$

ДокАЗАТЕЛЬСТво. Пространство $X$ есть фильтрованное пространство с фильтрацией

$$
X=\bigcup_{k} X^{(k)}, \quad X^{(k+1)} \supset X^{(k)},
$$

где $X^{(k)}$ порождено векторами $X_{I}$ с $|I|=k$. Пространства $X^{(k)}$ конечномерны. Аналогично для $D\left(\mathbb{Z}_{p}\right)$ имеет место фильтрация конечномерными подпространствами

$$
D\left(\mathbb{Z}_{p}\right)=\bigcup_{k} D_{k}\left(\mathbb{Z}_{p}\right), \quad D_{k+1}\left(\mathbb{Z}_{p}\right) \supset D_{k}\left(\mathbb{Z}_{p}\right)
$$

где $D_{k}\left(\mathbb{Z}_{p}\right)$ порождено функциями $\theta_{k}(x-I)$ с $|I|=k$.

Для доказательства леммы достаточно показать, что $\phi$ есть изоморфизм для отображений $\phi: X^{(k)} \rightarrow D_{k}\left(\mathbb{Z}_{p}\right)$. По определению $D_{k}\left(\mathbb{Z}_{p}\right)$ является конечномерным евклидовым пространством, порожденным функциями $\theta_{|I|}(x-I)$ с $|I|=k$. Функции $\theta_{|I|}(x-I)$ удовлетворяют соотношению

$$
\sum_{i=0}^{p-1} \theta_{|I i|}(x-I i)=\theta_{|I|}(x-I)
$$


и имеют скалярные произведения

$$
\left(\theta_{k}(x-I), \theta_{k}(x-J)\right)=p^{-|I|} \delta_{I J}
$$

Пространство $X^{(k)}$ порождено $X_{I}$ с $|I|=k$. Векторы $X_{I}$ удовлетворяют соотношению

$$
X_{I}=p^{-1} \sum_{j=0}^{p-1} X_{I j},
$$

которое мы выводим из (6) следующим образом:

$$
\begin{aligned}
X_{I} & =\sum_{k=0}^{\infty} \lambda^{k}\left(\frac{1}{p} \sum_{i=0}^{p-1} A_{i}^{\dagger}\right)^{k} \lambda^{|I|} A_{I}^{\dagger} \Omega+\sum_{l=1}^{\infty} \lambda^{-l}\left(\sum_{i=0}^{p-1} A_{i}\right)^{l} \lambda^{|I|} A_{I}^{\dagger} \Omega= \\
& =p^{-1} \sum_{j=0}^{p-1} \sum_{k=0}^{\infty} \lambda^{k}\left(\frac{1}{p} \sum_{i=0}^{p-1} A_{i}^{\dagger}\right)^{k} \lambda^{|I j|} A_{I j}^{\dagger} \Omega+\lambda^{|I|} A_{I}^{\dagger} \Omega+\sum_{l=1}^{\infty} \lambda^{-l}\left(\sum_{i=0}^{p-1} A_{i}\right)^{l} \lambda^{|I|} A_{I}^{\dagger} \Omega= \\
& =p^{-1} \sum_{j=0}^{p-1} \sum_{k=0}^{\infty} \lambda^{k}\left(\frac{1}{p} \sum_{i=0}^{p-1} A_{i}^{\dagger}\right)^{k} \lambda^{|I j|} A_{I j}^{\dagger} \Omega+p^{-1} \sum_{j=0}^{p-1} \sum_{l=1}^{\infty} \lambda^{-l}\left(\sum_{i=0}^{p-1} A_{i}\right)^{l} \lambda^{|I j|} A_{I j}^{\dagger} \Omega= \\
& =p^{-1} \sum_{j=0}^{p-1} X_{I j} .
\end{aligned}
$$

Векторы $X_{I}$ имеют следующие скалярные произведения для $|I|=|J|$ :

$$
\left(X_{I}, X_{J}\right)=p^{|I|} \delta_{I J}
$$

Сравнивая (15), (16) и (17), (18), мы получаем утверждение леммы.

СлЕДСтвиЕ 1. Перенормированное скалярное произведение $\left(X_{I}, X_{J}\right)$ CKC $X_{I}, X_{J} \in X$ равно интегралу по р-адическому диску по мере Хаара $\mu$ :

$$
\begin{aligned}
\left(X_{I}, X_{J}\right) & =p^{|I|+|J|} \int_{\mathbb{Z}_{p}} \theta_{|I|}(x-I) \theta_{|J|}(x-J) \mu(d x)= \\
& =\left(\frac{\theta_{|I|}(x-I)}{\left\|\theta_{|I|}(x-I)\right\|^{2}}, \frac{\theta_{|J|}(x-J)}{\left\|\theta_{|J|}(x-J)\right\|^{2}}\right)_{L^{2}} \cdot
\end{aligned}
$$

Лемма 3. Изоморфизм ф порождает вложсение $\phi^{\prime}$ пространства СКС $X^{\prime}$ в пространство обобщенных функций на р-адическом диске $D^{\prime}\left(\mathbb{Z}_{p}\right)$ :

$$
\phi^{\prime}(\Psi)=\Psi \circ \phi^{-1}
$$


ДокАЗАТЕЛЬСтво. Мы должны проверить, что для произвольного ненулевого CKC $\Psi$ функционал $\Psi \circ \phi^{-1}$ является ненулевым непрерывным линейным функционалом над $D\left(\mathbb{Z}_{p}\right)$.

Согласно (8) и (14) имеем

$$
\left(\Psi \circ \phi^{-1}, \theta_{|I|}(x-I)\right)=\Psi_{I}
$$

Поскольку для любого ненулевого СКС $\Psi$ по крайней мере один коэффициент $\Psi_{I}$ является ненулевым, это доказывает, что функционал $\Psi \circ \phi^{-1}$ ненулевой.

Топология в $D\left(\mathbb{Z}_{p}\right)$ определяется следующим образом (см. [3]). Пространство $D\left(\mathbb{Z}_{p}\right)$ есть

$$
D\left(\mathbb{Z}_{p}\right)=\bigcup_{k} D_{k}\left(\mathbb{Z}_{p}\right)
$$

где $D_{k}\left(\mathbb{Z}_{p}\right)$ - линейная оболочка $\theta_{j}(x-I), j \leqslant k$, и последовательность $\left\{\phi_{j}\right\}$ в $D\left(\mathbb{Z}_{p}\right)$ сходится, если все $\phi_{j} \in D_{k}\left(\mathbb{Z}_{p}\right)$ для любого $j$ и некоторого $k$ и функции $\phi_{j} \rightarrow 0$ однородно. Каждое из пространств $D_{k}\left(\mathbb{Z}_{p}\right)$ есть нормированное пространство (с $C$-нормой, равной верхней грани по $p$-адическому диску от модуля функции).

Из формулы (8) следует, что $\Psi \circ \phi^{-1}$ есть ограниченный функционал на $D_{k}\left(\mathbb{Z}_{p}\right)$ с нормой

$$
\left\|\Psi \circ \phi^{-1}\right\|_{D_{k}\left(\mathbb{Z}_{p}\right)}=\max _{|I| \leqslant k}\left|\Psi_{I}\right|
$$

Следовательно, функционал $\Psi \circ \phi^{-1}$ непрерывен на $D\left(\mathbb{Z}_{p}\right)$, что завершает доказательство леммы.

Важным примером обобшенной функции является $\delta$-функция. Введем когерентное состояние, отвечающее $\delta$-функции. Рассмотрим бесконечную последовательность $I=$ $i_{0} \ldots i_{k} \ldots, i_{j}=0, \ldots, p-1$, и соответствуюшее $p$-адическое число $I=\sum_{k=0}^{\infty} i_{k} p^{k}$. Обозначим $I_{k}=i_{0} \ldots i_{k-1}$. Введем СКС $\delta_{I}$ вида

$$
\delta_{I}=\sum_{k=0}^{\infty} \lambda^{k} A_{I_{k}}^{\dagger} \Omega .
$$

ПримеР. Отображение $\phi^{\prime}$ переводит СКC $\delta_{I}$ в $\delta$-функцию:

$$
\phi^{\prime}\left(\delta_{I}\right)=\delta(x-I)
$$

ДокАЗАТЕЛЬСТво следует из формул (8) и (19).

Лемма 4. Вложсение пространства СКС $X^{\prime}$ в пространство обобщенных функиий на р-адическом диске $D^{\prime}\left(\mathbb{Z}_{p}\right)$, построенное в лемме 3 , сюрвективно ( вательно, является изоморфизмом линейных пространств). 
ДокАЗАТЕльСтво. Для доказательства леммы достаточно построить СКС, которое при применении к обратному образу индикатора произвольного $p$-адического диска в $\mathbb{Z}_{p}$ даст произвольное (с учетом соотношения, следуюшего из линейности функционала и того факта, что индикатор диска равен сумме индикаторов поддисков) комплексное число. Это следует из формулы (20):

$$
\left(\Psi, \phi^{-1} \theta_{|I|}(x-I)\right)=\Psi_{I},
$$

где $\Psi_{I}$ есть произвольный набор комплексных чисел, удовлетворяющих соотношению (5), выражающему свойство линейности, примененное к индикаторам поддисков:

$$
\left(\Psi, \phi^{-1} \theta_{|I|}(x-I)\right)=\sum_{i=0}^{p-1}\left(\Psi, \phi^{-1} \theta_{|I i|}(x-I i)\right) .
$$

Это завершает доказательство леммы.

СЛЕДСТВИЕ 2. Отображения $\phi, \phi^{\prime}$ (которье являются изоморфизмами линейных пространств) позволяют перенести топологию $D\left(\mathbb{Z}_{p}\right)$ и $D^{\prime}\left(\mathbb{Z}_{p}\right)$ на пространства $X$ и $X^{\prime}$, соответственно. Эта прочедура делает отображсения $\phi и \phi^{\prime}$ изоморфизмами топологических векторных пространств.

\section{4. ОСНАШЕННОЕ ГИЛЬБЕРТОВО ПРОСТРАНСТВО СВОБОДНЫХ КОГЕРЕНТНЫХ СОСТОЯНИЙ}

Леммы 2-4 подсказывают следуюшее определение.

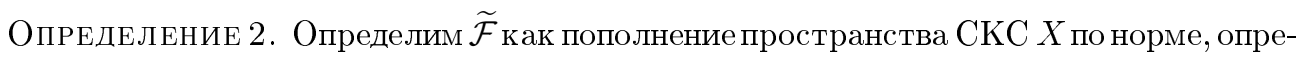
деленной перенормированным скалярным произведением.

Пространство $\widetilde{\mathcal{F}}$ есть гильбертово пространство по отношению к перенормированному скалярному произведению.

Лемма 5. Гильбертово пространство $\widetilde{\mathcal{F}}$ лежит в пространстве $C K C X^{\prime}: \widetilde{\mathcal{F}} \subset$ $X^{\prime}$

ДокаЗАТЕЛЬСтво. Для $\Psi \in \widetilde{\mathcal{F}}, \Psi=\lim _{n \rightarrow \infty} \Psi^{(n)}, \Psi^{(n)} \in X$, рассмотрим произведение (11). Принимая во внимание (17), получим

$$
\left(\Psi, X_{I}\right)=p^{-1} \sum_{i=0}^{p-1}\left(\Psi, X_{I i}\right),
$$

откуда следует, что $\Psi_{I}$, где $\Psi_{I}=\left(\Psi, X_{I}\right) p^{-|I|}$, удовлетворяет (5). Следовательно,

$$
\widetilde{\Psi}=\sum_{I} \lambda^{|I|} \Psi_{I} A_{I}^{\dagger} \Omega
$$

есть СКС в $X^{\prime}$, удовлетворяющее равенствам

$$
\left(\widetilde{\Psi}, X_{I}\right)=\left(\Psi, X_{I}\right)=p^{|I|} \Psi_{I}
$$

откуда следует, что $\widetilde{\Psi}=\Psi$. Это завершает доказательство леммы. 
Лемма 6. Отображсение $j: X \rightarrow \widetilde{\mathcal{F}}$ в (21) (см. ниже) есть непрерывное вложение с плотным образом.

ДоКАЗАТЕЛЬСТво. Из формулы (8) следует, что ненулевое СКС в $X$ имеет ненулевую норму в $\widetilde{\mathcal{F}}$. Следовательно, $j$ является вложением.

Пусть $\left\{\Phi^{(n)}\right\}$ есть сходящаяся (в топологии, индуцированной из $\left.D\left(\mathbb{Z}_{p}\right)\right)$ последовательность в $X$. Чтобы проверить непрерывность вложения $j$, нужно показать, что последовательность $\left\{\Phi^{(n)}\right\}$ фундаментальна в $\widetilde{\mathcal{F}}$. По определению топологии в $D\left(\mathbb{Z}_{p}\right)$ сушествует $k$ такое, что $\left\{\Phi^{(n)}\right\} \subset X^{(k)}$. Следовательно, каждьй вектор $\Phi^{(n)}$ есть конечная линейная комбинация векторов $X_{I},|I|=k$, и сходимость последовательности $\left\{\Phi^{(n)}\right\}$ сводится к сходимости конечного числа коэффициентов в разложении по $X_{I}$. Согласно (8) получаем, что $\left\{\Phi^{(n)}\right\}$ есть фундаментальная последовательность в $\widetilde{\mathcal{F}}$, что доказывает непрерывность $j$. Это завершает доказательство леммы.

Суммируя леммы 5 и 6 , мы получаем следующую теорему.

\section{Teоpema 1. Пространство СKC}

$$
X \stackrel{i}{\rightarrow} \widetilde{\mathcal{F}} \stackrel{j}{\rightarrow} X^{\prime}
$$

является оснащенным гильбертовым пространством.

Напомним, что оснашенное гильбертово пространство есть тройка пространств

$$
A \stackrel{i}{\rightarrow} \mathcal{H} \stackrel{j}{\rightarrow} A^{*}
$$

где $\mathcal{H}$ есть гильбертово пространство, $A$ и $A^{*}$ являются взаимно сопряженными топологическими векторными пространствами, отображения $i: A \rightarrow \mathcal{H}$ и $j: \mathcal{H} \rightarrow A^{*}$ суть непрерывные вложения, образ $i$ плотен в $\mathcal{H}$, отображения $i$ и $j$ взаимно сопряжены в следуюшем смысле:

$$
(i a, h)=(a, j h), \quad a \in A, \quad h \in \mathcal{H} .
$$

Сравним оснашенные гильбертовы пространства CKC (21) и обобшенных функций на р-адическом диске:

$$
D\left(\mathbb{Z}_{p}\right) \stackrel{i^{\prime}}{\rightarrow} L^{2}\left(\mathbb{Z}_{p}\right) \stackrel{j^{\prime}}{\rightarrow} D^{\prime}\left(\mathbb{Z}_{p}\right)
$$

Получим следуюшую теорему, являюшуюся расширением теоремы, доказанной в [2].

ТЕОРема 2. Отображсение $\phi$, определенное как

$$
\phi: X_{I} \mapsto p^{|I|} \theta_{|I|}(x-I),
$$


расширяется до изоморфизма ф оснащенных гильбертовых пространств

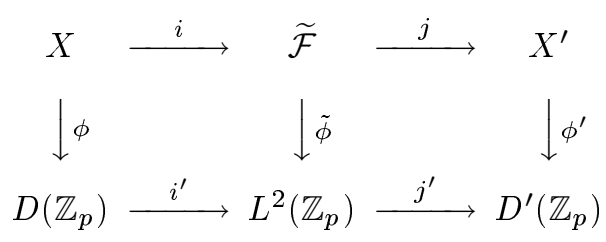

между оснащенным гильбертовым пространством СКС (с перенормированным спариванием) и оснащенным гильбертовым пространством обобщенных функций на р-адическом диске.

Здесь $\tilde{\phi}$ есть ограничение $\phi^{\prime}$ на $\widetilde{\mathcal{F}}$.

ДоКАЗАТЕЛЬСТво следует из лемм 2-4 и следствия 2. Изоморфизм между пространствами $\widetilde{\mathcal{F}}$ и $L^{2}\left(\mathbb{Z}_{p}\right)$ следует из того факта, что эти пространства являются пополнениями изоморфных пространств по совпадающей норме.

ЗАмЕчАниЕ. Определение (2) пространства СКС

$$
(A-\lambda) \Psi=0, \quad A=\sum_{i=0}^{p-1} A_{i}
$$

можно интерпретировать как уравнение некоммутативной (или квантовой) плоскости $A=\lambda$ в некоммутативном пространстве с некоммутативными координатами $A_{i}, A_{i}^{\dagger}$. CKC $\Psi$ в этой картине соответствует обобщенной функции на некоммутативном пространстве с носителем на некоммутативной плоскости $A=\lambda$.

Теорема 2 означает, что пространство обобщенных функций на некоммутативной плоскости изоморфно, как оснашенное гильбертово пространство, пространству обобшенных функций на $p$-адическом диске, или, другими словами, некоммутативная плоскость эквивалентна $p$-адическому диску.

Отметим, что значение $\lambda=\sqrt{p}$ есть максимальное возможное значение $\lambda$. Для $\lambda>$ $\sqrt{p}$ любой вектор (3) имеет бесконечную норму и, следовательно, не лежит в гильбертовом пространстве.

Благодарности. Автор благодарен И.В. Воловичу и О. Г. Смолянову за обсуждения и важные замечания. Эта работа была частично поддержана INTAS (грант № YSF2002-160 F2), CRDF (грант № UM1-2421-KV-02), РФФИ (грант № 02-01-01084) и Президентским грантом поддержки научных школ (НШ 1542.2003.1). 


\section{Список литературы}

[1] С. В. Козырев. ТМФ. 1997. Т. 110. № 2. С. 334; q-alg/9701015.

[2] S. V. Kozyrev. Infin. Dimens. Anal. Quantum Probab. 1998. V. 1. № 2. P. 349; q-alg/9706020.

[3] В.С. Владимиров, И. В. Волович, Е. И. Зеленов. р-Адический анализ и математическая физика. М.: Наука, 1994.

[4] I. V. Volovich. Class. Q. Grav. 1987. V. 4. P. L83.

[5] P.G.O. Freund, M. Olson. Phys. Lett. B. 1987. V. 199. P. 186; V.S. Vladimirov, I. V. Volovich. Commun. Math. Phys. 1989. V. 123. P. 659; I. Ya. Aref'eva, B. Dragovic, P. Frampton, I. V. Volovich. Mod. Phys. Lett. A. 1991. V. 6. P. 4341; В. C. Владимиров. УМH. 1989. T. 43. C. 17.

[6] A. Khrennikov. p-Adic Valued Distributions in Mathematical Physics. Dordrecht: Kluwer, 1994.

[7] S. Albeverio, A. Khrennikov. Int. J. Mod. Phys. 1998. V. 10. № 13/14. P. 1665; A. N. Kochubei. Additive and multiplicative fractional differentiations over the field of $p$-adic numbers. In: p-Adic Functional Analysis. Proc. of the 4th Int. Conf. (Nijmegen, Netherlands, June 3-7, 1996). Lect. Notes Pure Appl. Math. V. 192. Eds. W.H. Schikhof, C. Perez-Garcia, J. Kakol. New York: Dekker, 1997. P. 275.

[8] V.A. Avetisov, A.H. Bikulov, S. V. Kozyrev. J. Phys. A. 1999. V. 32. P. 8785; cond-mat/9904360; G. Parisi, N. Sourlas. European Phys. J. B. 2000. V. 14. P. 535; cond-mat/9906095.

[9] С. В. Козырев. Изв. РАН. Сер. матем. 2002. Т. 66. № 2. С. 149; math-ph/0012019.

[10] S. V. Kozyrev. The noncommutative replica approach. cond-mat $/ 0110238$.

[11] I. Ya. Aref'eva, I. V. Volovich. Nucl. Phys. B. 1996. V. 462. P. 600; R. Gopakumar, D. Gross. Nucl. Phys. B. 1995. V. 451. P. 379; M.R. Douglas, M. Li. Phys. Lett. B. 1995. V. 348. P. 360 .

[12] L. Accardi, Y. G. Lu. Commun. Math. Phys. 1996. V. 180. P. 605; L. Accardi, Y. G. Lu, I. V. Volovich. Quantum Theory and its Stochastic Limit. Berlin: Springer, 2002; Interacting Fock Spaces and Hilbert Module Extensions of the Heisenberg Commutation Relations. Kyoto: Publ IIAS, 1997; Л. Аккарди, И. В. Волович, С. В. Козырев. ТМФ. 1998. Т. 116. № 3. C. 401; D. Voiculescu, K.J. Dykema, A. Nica. Free Random Variables. CRM Monograph Series. V. 1. Providence, RI: AMS, 1992; M. Bozejko, R. Speicher. Commun. Math. Phys. 1991. V. 137. P. 519. 